\title{
BMJ Open Short-term morbidity and mortality following radical cystectomy: a systematic review
}

\author{
Sophia Liff Maibom (1) , ,,2 Ulla Nordström Joensen, ${ }^{1,2}$ Alicia Martin Poulsen, ${ }^{3}$ \\ Henrik Kehlet, ${ }^{2,4}$ Klaus Brasso, ${ }^{1}$ Martin Andreas Røder ${ }^{1,2}$
}

To cite: Maibom SL, Joensen UN, Poulsen AM, et al. Short-term morbidity and mortality following radical cystectomy: a systematic review. BMJ Open 2021;11:e043266. doi:10.1136/ bmjopen-2020-043266

- Prepublication history and additional supplemental material for this paper are available online. To view these files, please visit the journal online (http://dx.doi.org/10.1136/ bmjopen-2020-043266).

Received 29 July 2020

Revised 03 March 2021

Accepted 19 March 2021
Check for updates

(C) Author(s) (or their employer(s)) 2021. Re-use permitted under CC BY-NC. No commercial re-use. See rights and permissions. Published by BMJ.

For numbered affiliations see end of article.

Correspondence to Sophia Liff Maibom; d034920@dadlnet.dk

\section{ABSTRACT}

Objective To study short-term ( $<90$ days) morbidity and mortality following radical cystectomy (RC) for bladder cancer and identify modifiable risk factors associated with these.

Design Systematic review.

Methods The systematic review was conducted according to the Preferred Reporting Items for Systematic Reviews and Meta-Analyses guidelines. PubMed and EMBASE were searched for relevant papers on 11 June 2019 and rerun on 27 May 2020. Studies reporting complications, reoperations, length of stay and mortality within 90 days were included. Studies were reviewed according to criteria from the Oxford Centre for EvidenceBased Medicine and the quality of evidence was assessed using the Newcastle-0ttawa Scale.

Results The search retrieved 1957 articles. Sixty-six articles were included. The quality of evidence was poor to good. Most studies were retrospective, and no randomised clinical trials were identified. Of included studies a median of 6 Martin criteria for reporting complications after surgery were fulfilled. The Clavien-Dindo classification for grading complications was most frequently used. The weighted overall complication rate after RC was $34.9 \%$ (range 28.8-68.8) for in-house complications, $39.0 \%$ (range 27.3-80.0) for 30-day complications and $58.5 \%$ (range $36.1-80.5$ ) for 90 -day complications. The most common types of complications reported were gastrointestinal (29.0\%) and infectious (26.4\%). The weighted mortality rate was $2.4 \%$ (range $0.9-4.7$ ) for in-house mortality, $2.1 \%(0.0-3.7)$ for 30 -day mortality and $4.7 \%$ (range $0.0-7.0$ ) for 90-day mortality. Age and comorbidity were identified as the best predictors for complications following RC.

Conclusion Short-term morbidity and mortality are high following RC. Reporting of complications is heterogeneous and the quality of evidence is generally low. There is a continuous need for randomised studies to address any intervention that can reduce morbidity and mortality following $\mathrm{RC}$.

PROSPERO registration number 104937.

\section{INTRODUCTION}

Radical cystectomy (RC) with pelvic lymph node dissection and urinary diversion is the preferred treatment for non-metastatic muscle-invasive bladder cancer (BC), and for

\section{Strengths and limitations of this study}

- This systematic review can provide as a reference paper for future studies and when measuring quality of care.

- This systematic review emphasises the continuous need to identify and moderate risk factors for complications and optimise postoperative management plans to reduce both morbidity and mortality associated with radical cystectomy.

- This review is limited by heterogeneity in outcome measures of morbidity with a lack of clear definitions of surgical complications making a direct comparison between studies difficult.

some cases of high-risk non-muscle-invasive $\mathrm{BC}$, in patients fit for major surgery. ${ }^{1} \mathrm{RC}$ is a comprehensive procedure that involves surgery to several organ systems and as a result it is associated with high postoperative morbidity and mortality. Attempts have been made over the years to reduce postoperative complications such as the introduction of Enhanced Recovery After Surgery (ERAS) programmes. However, addressing morbidity and mortality associated with RC across surgical cohorts remains important for preoperative counselling, planning of treatment, identification of modifiable risk factors to reduce morbidity and mortality, future clinical trial design and for assessment of surgical quality. Several measures of morbidity are clinically important such as complication rate, reoperation rate, length of stay (LOS), readmission rate and mortality. In this paper, we conducted a contemporary systematic review of the prevalence of short-term $(<90$ days) morbidity and mortality following $\mathrm{RC}$ for BC.

\section{METHODS}

Search strategy and study selection

This systematic review was performed in accordance with the Preferred Reporting 


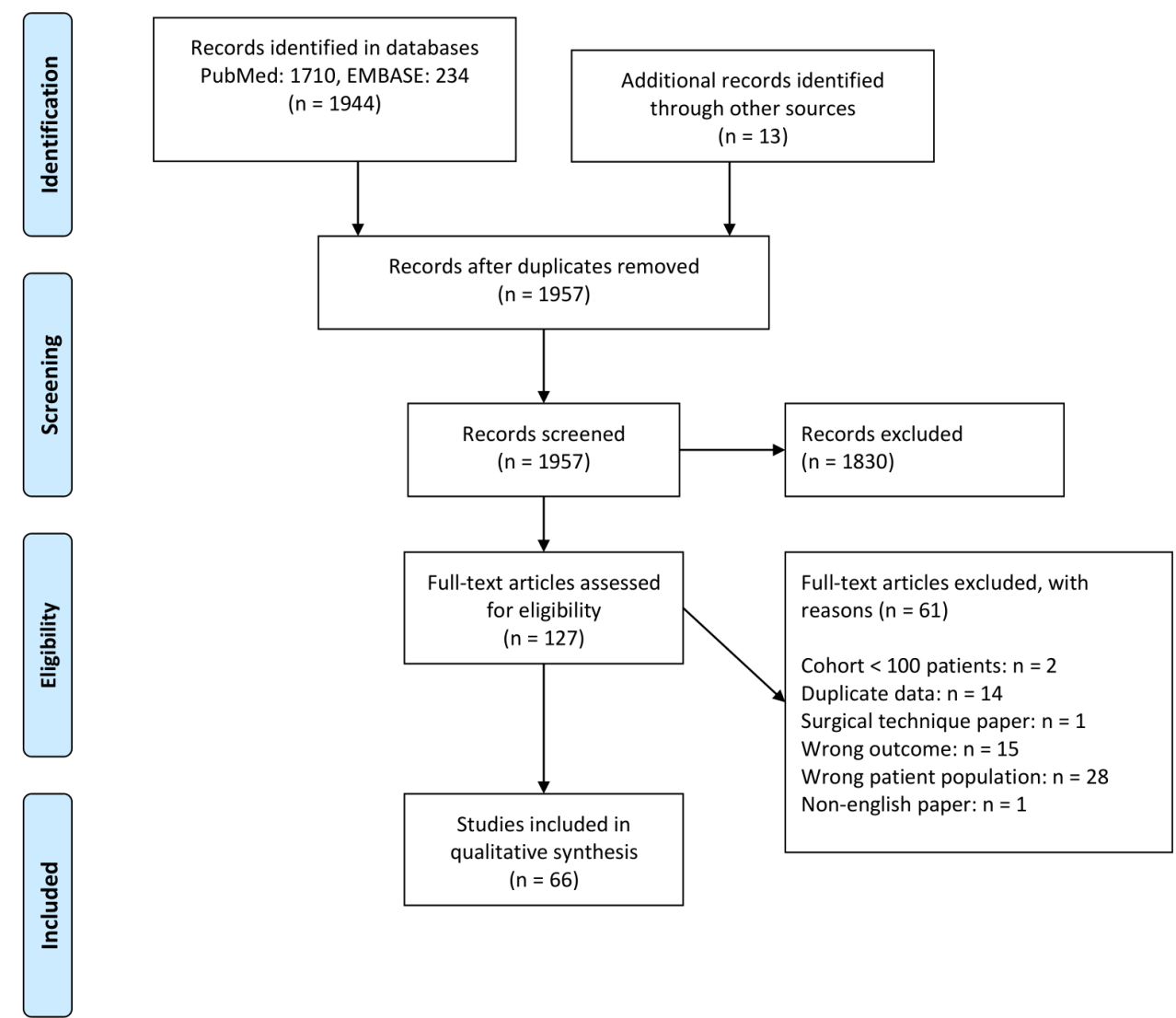

Figure 1 Preferred Reporting Items for Systematic Reviews and Meta-Analyses (PRISMA) flow chart. From: Moher D et al. ${ }^{105}$

Items for Systematic Reviews and Meta-Analyses guidelines. ${ }^{2}$ A published protocol (PROSPERO) with prespecified outcomes, inclusion criteria and search strategy is accessible online.

A systematic literature search in PubMed and EMBASE was conducted on 11 June 2019 and rerun on 27 May 2020. A search string was created with the help of an information specialist (online supplemental appendix 1).

Articles were screened in a two-stage selection process. In the first stage, two authors (SLM and MAR) reviewed the abstracts. All prospective and retrospective studies on short-term ( $<90$ days) morbidity and mortality after RC were included. Trials with less than 100 participants, indications for cystectomy other than BC, extended procedure (eg, nephroureterectomy), salvage/palliative cystectomy, organ sparing cystectomy (eg, partial cystectomy, prostate sparing cystectomy, vaginal sparing cystectomy, seminal vesicles sparing cystectomy), selected patient group (eg, certain age groups, women only), feasibility studies, surgical technique-only papers, animal series and studies not published in English were excluded. Conference papers, case reports, book chapters, review papers, editorials, comments, letters to the editors and abstracts were also excluded. When in doubt, studies were maintained for further review. In the second stage, the full text of all included articles was obtained and read by the same two authors. An agreement was reached through consensus using Covidence Systematic Review software. ${ }^{3}$ Any disagreement was resolved by discussion and the final decision was based on a consensus. In case of duplicate data/study the following criteria were applied in the selection: (1) outcome (studies reporting on complications were prioritised over LOS, mortality), (2) size of the cohort (larger studies were prioritised over smaller studies), (3) methodology (prospective studies were prioritised over retrospective studies and extraction of data from medical/hospital records over record linkage (eg, International Classification of Diseases codes in a database)), and (4) study period (studies with the most recent study period were prioritised).

\section{Data extraction and quality assessment}

The following data were extracted from all studies where possible: first author, data source (eg, single centre, multicentre, database), institution/country of origin, study period, year of publication, number of cases, study design, length of follow-up, the classification system used for grading complications, use of fast track/ERAS protocol, demographics (age, gender, body mass index, Charlson Comorbidity Index (CCI), American Society of Anesthesiologists (ASA) score, pT-stage, N-stage, neoadjuvant therapy, previous radiation therapy, prior abdominal/ pelvic surgery), outcomes (urinary diversion, number of total complications, complication rate, segregated complications, complication reasons, mortality rate, LOS, reoperations, risk factors for outcomes).

The quality of reporting complications was estimated using the Martin criteria providing a score from 0 to $10 .^{4}$ 

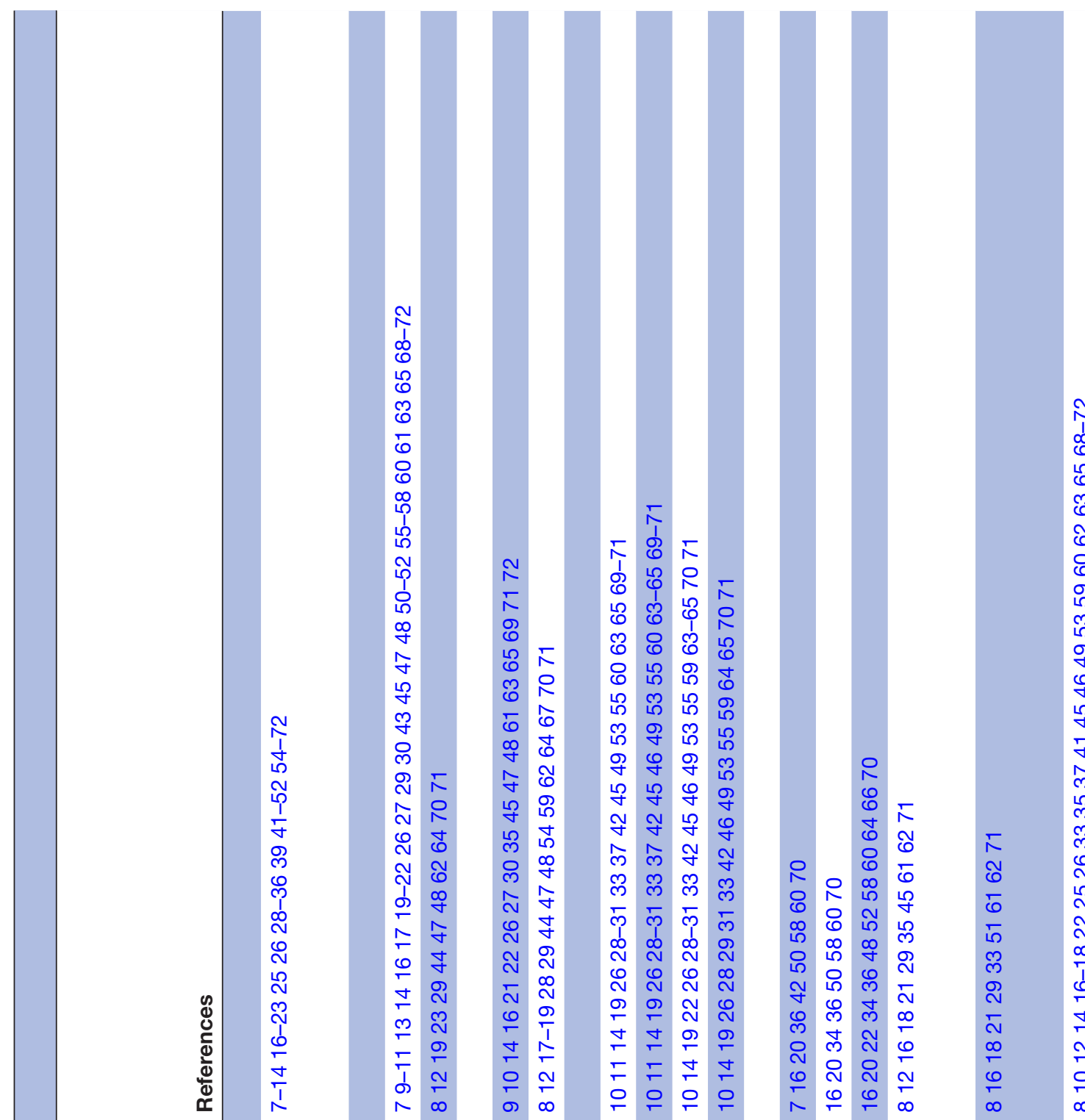

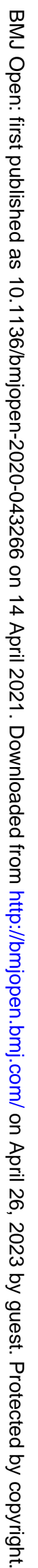

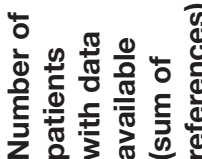

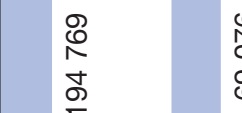

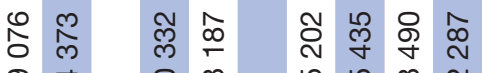

8 우

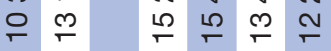

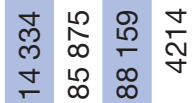

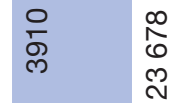

ำ

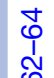

8

เับ

市

约

ঙ

г

ल

ले

लn

p

กิ

กิ

$\frac{1}{1}$

$\underset{\infty}{\frac{m}{5}}$

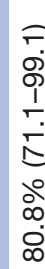
T.

कิ

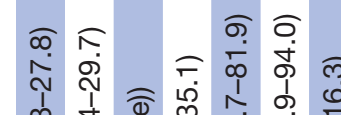

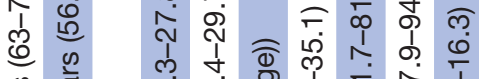

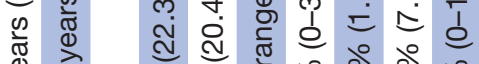

ㅇำ

官

m 0

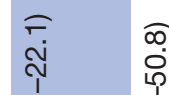



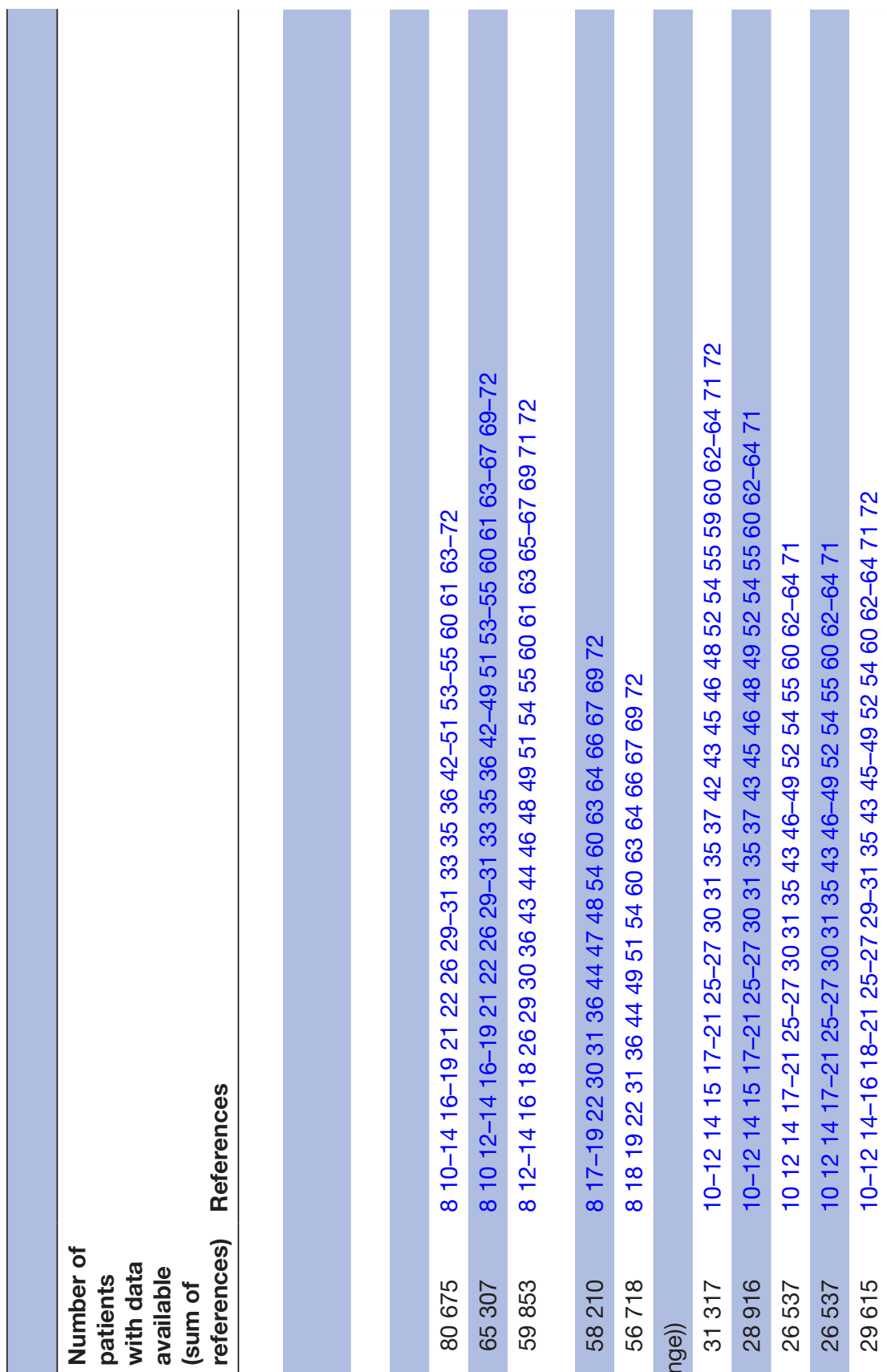

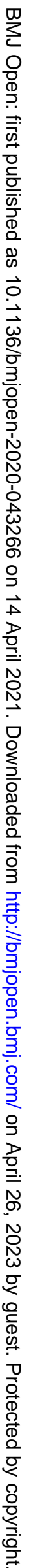

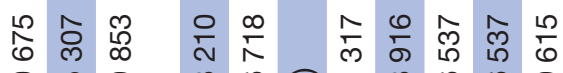
$\infty$ 뉴

冓

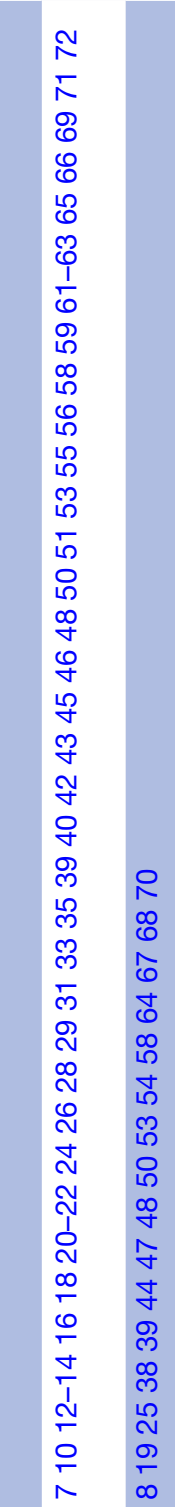

$\frac{\pi}{4}$

营

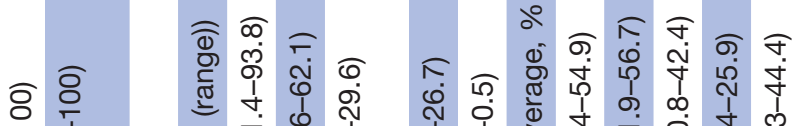

I d a

d

당

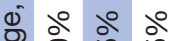

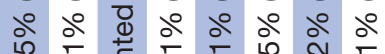

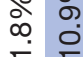

娄

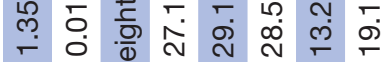

(1)

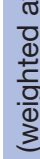

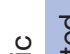

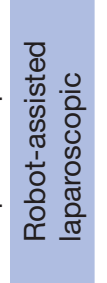

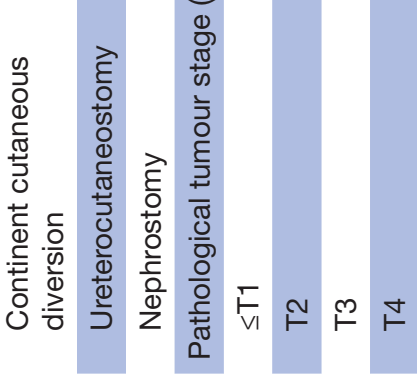


Furthermore, the level of evidence was rated according to criteria from the Oxford Centre for Evidence-Based Medicine. ${ }^{5}$ The methodological quality of the studies was assessed using the Newcastle-Ottawa Scale (NOS) for observational comparative studies. ${ }^{6}$

\section{Outcome measures}

The primary outcome was the overall complication rate: the number of patients with one or more complication(s) within 90 days after $\mathrm{RC}$ regardless of the classification system used. Secondary outcomes were the following: rate of graded complications according to severity grade used; frequencies of types of complications; LOS, reoperation rate; mortality rate; and risk factors for the development of outcomes of morbidity (eg, complications, death, reoperations).

\section{Statistical analysis}

Descriptive statistics were used. A weighted average and range were calculated for all rates. A meta-analysis on risk factors for morbidity was not possible due to the high heterogeneity of reporting in the multivariate analysis across studies.

\section{Patient and public involvement}

No patients were involved in conducting this review.

\section{RESULTS}

The literature search retrieved 1957 articles after removing duplicates. Of these, 66 studies met the inclusion and exclusion criteria. ${ }^{7-72}$ The process is outlined in figure 1 .

\section{Characteristics of included studies}

The characteristics of the included studies are summarised in table 1 . Twenty-nine studies $(43.9 \%)$ were single-centre studies and 37 studies $(56.1 \%)$ were register or multicentre database studies. Most studies (71.2\%) were retrospective, retrospective studies of prospectively maintained databases $(12.1 \%)$ or combined retrospective and prospective studies $(4.5 \%)$. Only eight $(12.1 \%)$ were purely prospective surgical series. Patients were operated in the period 1990-2018. Of included studies, only two reported that an ERAS protocol was used for the entire cohort, ${ }^{1648}$ and in six studies an ERAS protocol was used in a part of the cohort. ${ }^{33} 3942676972$ In the rest of the included studies, an ERAS protocol was not used, or the authors did not report on perioperative care.

\section{Complications}

Fifty-two studies reported on short-term complications as outlined in table 2 . The most frequently reported follow-up period was 90 days. Three studies reporting short-term complications did not state the exact follow-up period and were therefore excluded from the complication rate analysis. ${ }^{33} 4067$ During the primary hospitalisation, the overall complication rate was $34.9 \%$ (28.8-68.8). The complication rate increased with longer follow-up to $39.0 \%(27.3-80.0)$
30 days and $58.5 \%$ (36.1-80.5) 90 days postoperatively. Minor complications accounted for $40.0 \%$ (19.9-77.4) and $38.2 \%$ (19.0-80.8) of the complications reported at 30 and 90 days of follow-up, respectively. Major complications after RC occurred in $15.5 \%$ (4.9-24.8) and 16.9\% (13.4-32.0) of patients after 30 and 90 days, respectively. Rates of complications according to the Clavien-Dindo classification and reoperations are further outlined in table 2.

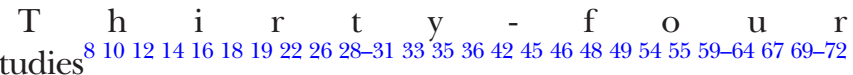
classified complications according to the Clavien-Dindo classification, ${ }^{73}$ six $^{913} 17434451$ studies classified complications as minor and major complications, three studies ${ }^{21} 27$ used the Memorial Sloan Kettering Cancer Center-modified Clavien-Dindo classification, ${ }^{61}$ one study ${ }^{65}$ used Common Terminology Criteria for Adverse Events ${ }^{74}$ and nine studies $^{2325} 32394047505358$ did not use any system for grading complications.

\section{Type of complications}

Twenty-one studies reported on types of 90-day complications (table 3). Gastrointestinal (GI) (29.0\%) and infectious (26.4\%) complications were the most frequent.

\section{Mortality}

Fifty-three studies were included in the mortality analysis (table 4). The weighted average for the in-hospital mortality rate was $2.4 \%(0.9-4.7)$, the 30 -day mortality rate $2.1 \%(0.0-$ 3.7 ) and the 90 -day mortality rate $4.7 \%$ (0.0-7.0). A total of 17 of 53 studies reporting on mortality stated the causes of death with 183 deaths reported. ${ }^{101621222629-31354552535761646971}$ The most frequent cause of death was cardiopulmonary events accounting for $30 \%$ followed by progression of BC (15\%) and sepsis (11\%).

\section{Quality of studies}

Only three of the included studies ${ }^{226175}$ met 10 of 10 Martin criteria (online supplemental appendix 2). The median number of fulfilled Martin criteria was 6 (range 2-10). The only criterion fulfilled by all studies was defining the method of accruing data. The level of evidence according to criteria from the Oxford Centre for Evidence-Based Medicine was rated as 3 or 4 . The methodological quality across studies was 'poor' to 'good' assessed using the NOS (online supplemental appendix 2).

\section{DISCUSSION}

We systematically reviewed the literature to accurately describe short-term morbidity and mortality following RC and identify modifiable risk factors associated with these. The aim was to identify factors that could form the basis for the design of future randomised trials on postoperative interventions that can reduce the risk of complications.

\section{Main results}

$\mathrm{RC}$ is an extensive urological procedure and associated with a high risk of short-term minor and major morbidity. Mortality within 90 days of primary surgery is not 
Table 2 Complications and reoperations

\begin{tabular}{|c|c|c|c|}
\hline Outcome & $\begin{array}{l}\text { Complication rate, } \\
\text { weighted average } \\
\text { (\%-range) }\end{array}$ & $\begin{array}{l}\text { Number of } \\
\text { patients with data } \\
\text { available (sum of } \\
\text { references) }\end{array}$ & References \\
\hline $\begin{array}{l}\text { 30-day complication } \\
\text { rate }\end{array}$ & $39.0 \% \dagger(27.3-80.0)$ & 19160 & 91823283043444647515355 60-62 70-72 \\
\hline CD grade II & $29.8 \%(20.6-52.5)$ & 1291 & 30354570 \\
\hline CD grade III $a+b$ & $6.9 \%(5.6-14.4)$ & 8749 & 2830354570 \\
\hline CD grade IVa+b & $7.8 \%(0.7-11.0)$ & 8749 & 2830354570 \\
\hline CD grade $\mathrm{V}$ & $1.7 \%(0.0-2.1)$ & 8982 & 283035454670 \\
\hline $\begin{array}{l}90 \text {-day complication } \\
\text { rate }\end{array}$ & $58.5 \%$ ๆ (36.1-80.5) & 10625 & 81012141617212226 29-3142 484954 59-61 63-65 697172 \\
\hline CD grade I & $15.0 \%(4.0-31.6)$ & 4442 & 29305459616469 \\
\hline CD grade II & $38.9 \%(27.0-67.4)$ & 4442 & 2930545961646972 \\
\hline CD grade IIla+b & $20.5 \%(8.5-39.2)$ & 5548 & 29-31545961646972 \\
\hline $\mathrm{CD}$ grade IVa+b & $3.0 \%(0.2-8.5)$ & 5548 & 29-31545961646972 \\
\hline CD grade $V$ & $3.5 \%(0.1-3.9)$ & 55440 & 29-313648545961646972 \\
\hline $\begin{array}{l}\text { Minor } \\
\text { complication rate } \neq\end{array}$ & $38.2 \%(19.0-80.8)$ & 56955 & 81216172126313642 59-61 6369 \\
\hline
\end{tabular}

${ }^{*}$ One study ${ }^{25}$ did not report on overall complication rate.

†Three studies ${ }^{133545}$ did not report on overall complication rate.

¥Minor complications defined as Clavien-Dindo grades I-II, MSKCC grades 1-2 or minor complications.

§Major complications defined as Clavien-Dindo grades III-V, MSKCC grades 3-5 or major complications.

ПOne study ${ }^{36}$ did not report overall complication rate.

CD, Clavien-Dindo; MSKCC, Memorial Sloan Kettering Cancer Center.

negligible and occurs in $4.7 \%$ according to our review. Our systematic review underlines that complications occur in one in three patients during hospitalisation and that one in five patients have major complications during the first 30 days after RC. This emphasises the continuous need to identify and moderate risk factors for complications and optimise postoperative management plans to reduce both morbidity and mortality associated with RC.

Our analysis identified GI and infectious complications as the most frequently reported complications after RC. Overall, GI complications occurred in $29.0 \%$ with a postoperative ileus rate of $15.6 \%$. Urinary tract infections (UTI) were the most frequently occurring infectious complications occurring in $14.1 \%$ of patients.

\section{Risk factors for the development of Gl complications}

Based on the literature reviewed, it was not possible to identify the most important risk factors for GI complications and thus define whether these were potentially modifiable. The risk of ileus, which is often most clinically relevant, seems to be affected by many factors, most importantly increasing age. One study reported an increasing age as a statistically significant risk factor for ileus with an OR of 1.30 (95\% CI 1.1 to 1.5) per 10-year increase of age. ${ }^{23}$ This finding is supported by a large retrospective study (not included in this review) of 41 498 patients that found an increased OR of developing ileus with per 1-year increment in age (OR 1.012, 95\% CI 1.009 to $1.014, \mathrm{p}<0.05) .{ }^{76}$ The study also found that 
Table 3 Categories and type of 90-day complications

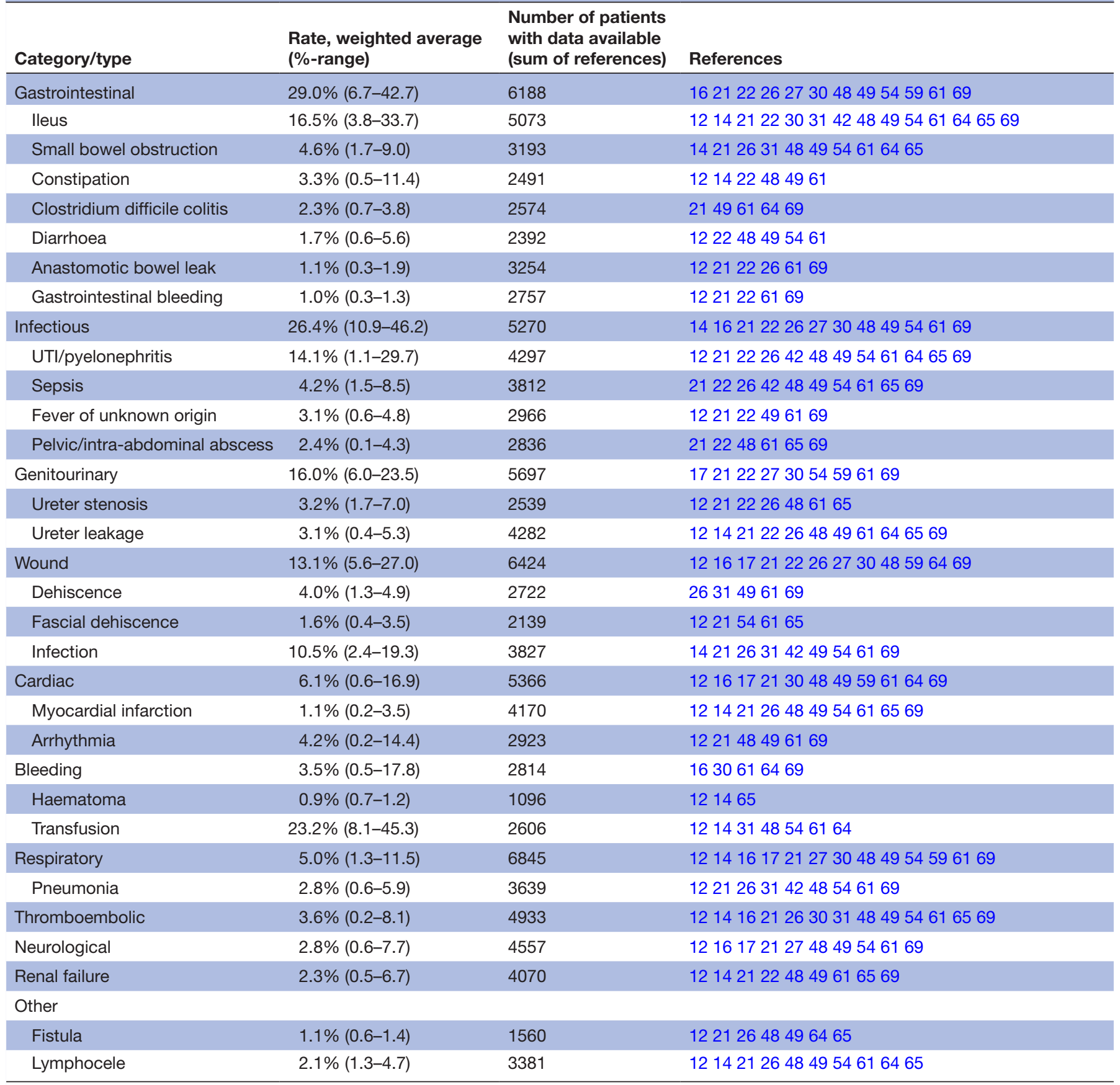

UTI, urinary tract infection.

the risk of ileus increased with several chronic conditions such as chronic pulmonary and neurological diseases. This underlines that reducing GI complication rates relies primarily on an overall medical assessment and that alternative treatment options should be considered in medically ill patients. Surgeons performing RC must be aware that poor general health status increases the risk of GI complications and entails a poor short-term outcome. Several studies of RC have promoted the implementation of ERAS protocols, which originate from colorectal surgery where ERAS reduces GI complications. In this review, no studies investigated the impact on GI complications with the use of an ERAS protocol versus a nonERAS protocol in the perioperative care. Generally, there is limited evidence for ERAS in an RC setting. ${ }^{77}$ Only the use of postoperative gum chewing, the use of alvimopan (a peripherally acting $\mu$-opioid receptor antagonist currently not available in Europe) and controlled administration of perioperative fluid management (goal-directed fluid therapy) to avoid both fluid excess and hypovolaemia have been shown to reduce GI complications after RC in randomised clinical trials (RCT) ${ }^{78-80}$ Comparative studies 
Table 4 In-hospital, 30-day and 90-day mortality

\begin{tabular}{llll}
\hline & $\begin{array}{l}\text { Mortality rate, } \\
\text { weighted } \\
\text { average } \\
\text { (\%-range) }\end{array}$ & $\begin{array}{l}\text { Number of } \\
\text { patients with } \\
\text { data available } \\
\text { (sum of } \\
\text { references) }\end{array}$ & References \\
\hline $\begin{array}{l}\text { In-hospital } \\
\text { mortality }\end{array}$ & $2.4 \%(0.9-4.7)$ & 87848 & 32415056586166 \\
\hline $\begin{array}{l}\text { 30-day } \\
\text { mortality }\end{array}$ & $2.1 \%(0.0-3.7)$ & 61299 & $7-913-1520242830333537384143-4651535760-6269-72$ \\
\hline $\begin{array}{l}\text { 90-day } \\
\text { mortality }\end{array}$ & $4.7 \%(0.0-7.0)$ & 108717 & $7810-1214161720-22242629-313336-3841484952545659-62646567-697172$ \\
\hline
\end{tabular}

indicate that omitting the nasogastric tube and mechanical bowel preparation result in lower GI complications after RC. ${ }^{81}{ }^{82}$ ERAS offers good practical guidelines, but the various elements such as early mobilisation, omitting pelvic drainage, perioperative body temperature monitoring and early oral diet are not studied individually but introduced in different modified versions with several components used together. Consequently, it is difficult to derive which factor has the largest impact on reducing GI complications. Four studies in this review investigated the impact on overall complications, but the results were conflicting. ${ }^{33} 396769$ A meta-analysis of ERAS protocols versus traditional protocols has found a faster return of bowel function and lower overall complication rate in the group managed on an ERAS compared with a standard of care protocol, but the overall level of evidence in RC remains low with regard to ERAS implementation. ${ }^{83} 84 \mathrm{~A}$ previous study described that only $20 \%$ of surgeons who endorse ERAS guidelines actually practised all interventions recommended by the ERAS society. ${ }^{85}$

\section{Risk factors for the development of infectious complications}

The anatomical reconstruction of the urinary tract with the use of bowel as urinary diversion following RC will naturally increase the risk of UTI, which can prolong LOS and is leading to readmittance. Only three of the included studies identified multivariable risk factors that were statistically significant predictors of infectious complications 30 and 90 days after RC. ${ }^{23} 2849$ Two studies found that continent reservoirs were associated with a higher risk of UTI compared with ileal conduits. Johnson et al found that any continent urinary diversion increased the risk of infectious complications compared with an incontinent urinary diversion (OR 1.68, $\mathrm{p}<0.001) .{ }^{28}$ Nazmy et al found that an Indiana pouch increased the risk of UTI compared with ileal conduit (OR 3.55, 95\% CI 1.33 to 9.44, $\mathrm{p}=0.01$ ); however, an orthotopic bladder substitute did not increase the risk of UTI compared with an ileal conduit. ${ }^{49}$ Other studies not included in this review investigating this association have shown conflicting results. ${ }^{86-89}$ Hollenbeck et al found preoperative bleeding disorder, poor functional status, preoperative acute renal failure and a $>10 \%$ weight loss preoperatively to be associated with an increased risk of UTI. ${ }^{23}$ In general, the comorbid patient may be at the highest risk for UTI. A large retrospective study of 1133 patients found that a CCI $>2$ was associated with a higher 90 -day postoperative UTI rate (OR $1.8,95 \%$ CI 1.1 to $2.9, \mathrm{p}=0.05$ ) compared with a CCI $0-2{ }^{86}$ It remains unclear if UTI can be prevented. No studies in this review investigated this question which in general is not well investigated. Pariser et al demonstrated that a change in prophylactic antibiotic protocol from a narrow to a broader coverage did not reduce the UTI rate, although the 30 -day risk of overall infectious complications was reduced following RC from $41 \%$ to $30 \%(\mathrm{p}=0.043) .{ }^{90}$ A population-based American study reported a lower infectious event rate when using a combination of antibiotic prophylaxis compared with a single-agent antibiotic (OR $0.79,95 \%$ CI 0.70 to 0.89 , $\mathrm{p}<0.001) .{ }^{91}$ The authors also investigated if extended antibiotic treatment $>24$ hours after RC decreased the risk of infectious complications, but no such association was found. Currently, international guidelines recommend that broad-spectrum antibiotics are used in the prophylactic regimen considering the local microbiological environment. ${ }^{92} 93$ However, RCTs addressing antibiotic prophylaxis regarding type, timing and duration for the risk of UTI are lacking and warranted.

\section{Risk factors for mortality}

In several studies, patient-related factors, age and comorbidity, were identified as the most important factors for mortality at index hospitalisation, as well as 30 and 90 days following surgery. ${ }^{11} 172427323841526668$

\section{Other risk factors}

In addition to patient-related factors, the impact of hospital volume, surgical experience and surgical technique has been addressed. Studies included in this review investigating the impact of hospital volume have shown conflicting results. ${ }^{15} 323437415666$ However, a previous meta-analysis of studies not all included in the present review found that the risk of postoperative mortality after RC was decreased by $45 \%$ when performed at a high-volume centre compared with a low-volume centre (pooled estimated effect OR 0.55, $95 \%$ CI 0.44 to 0.69$).{ }^{94}$ Studies also show that complications are reduced with both increasing hospital and surgeon volume. ${ }^{32} 36$ Unfortunately, the distinction 
between low volume versus high volume is not well defined and thus not comparable between studies. The European Association of Urology (EAU) MuscleInvasive and Metastatic Bladder Cancer Guideline Panel recommends RC to be performed at centres with at least $10 \mathrm{RC} /$ year and preferably $>20 \mathrm{RC} /$ year. $^{95}$ The surgical technique has been investigated in 12 of the included studies in this review. ${ }^{18202931333545486264}$ Open $\mathrm{RC}$ was compared with robotic-assisted $\mathrm{RC}$ in 10 of these non-randomised papers. In seven of these publications, a significantly reduced complication rate was found. However, five RCTs comparing open and robotic surgeries (not included in the review) did not find a difference in complication rates. ${ }^{96-100}$ All RCTs have been conducted with extracorporeal urinary diversion performed and it is speculated that robot-assisted RC with intracorporeal urinary diversion may have a lower complication rate compared with open RC. The question in hand is currently being studied in the ongoing iROC trial (robot-assisted radical cystectomy with intracorporeal urinary diversion versus open radical cystectomy) ${ }^{101}$.

\section{Limitations}

There are limitations of this review that must be addressed. Most of the included studies were retrospective which limits the clinical utility. It is important to notice that we excluded studies with less than 100 patients and studies investigating subgroups of patients such as certain age groups, types of urinary diversion or T-stages of BC. Since most RCTs on RC have less than 100 participants and often exclude patients with certain characteristics they were not included in this review.

\section{Challenges in the comparison of RC studies}

The difficulties of comparing RC studies are manifold. First, selection bias between cohorts must be expected. This is reflected by the wide range for the estimates of the weighted averages for ASA score and CCI (table 1). The selection of patients fit for RC is known to be associated with great variation among centres. ${ }^{102}$ Second, there was no standardised reporting of complications. Most used different classification systems for severity grade of complications with the Clavien-Dindo classification being the most frequent. Third, even when using a grading system as Clavien-Dindo with certain criteria, the scale can be interpreted differently or modified in some way. For example, some studies do not calculate blood transfusions as a complication even though it could be argued to be a grade II complication. Fourth, measures of morbidity can be defined differently across studies. For example, ileus is reported in up to $20 \%$ of patients undergoing RC. However, the reporting of ileus may be questioned as a previous systematic review found that ileus was defined differently across studies, and in the majority of included studies it was not defined at all. ${ }^{103}$ There is an increased focus on more uniform reporting of morbidity following $\mathrm{RC}$ and the
EAU has proposed authors to use quality criteria originally proposed by Martin et al. ${ }^{4104}$ In the present review, only three studies fulfilled all the Martin criteria. A previous non-systematic review from 2007 found no study reporting on complications after $\mathrm{RC}$ fulfilling all the Martin criteria. Lastly, publication bias must be emphasised as an important limitation.

We refrained from a meta-analysis of predictors of morbidity and mortality in this review as the number of studies investigating the same risk variable and outcome was too small for analysis. Identifying clinical predictors may aid in the prevention of postoperative morbidity and mortality. Currently, there is no risk assessment tool to predict postoperative outcomes after RC, and little correlation is found between the most frequently used risk-scoring systems (eg, CCI and ASA score) and postoperative outcomes. ${ }^{70}$ Nevertheless, in the included studies of this review, comorbidity was in multivariate logistic regression analysis consistently associated with a significantly increased OR of both complications ${ }^{12} 17222332495158606172$ and mortality. ${ }^{11172432415266}$ Surprisingly, there is a paucity of prospective studies studying the subjects of the most common complications after RC in order to identify clinical predictors of these. Furthermore, prospective randomised studies comparing different interventions/ regimens are lacking.

\section{CONCLUSION}

This review shows that $\mathrm{RC}$ is associated with a high risk of morbidity and mortality. However, with thorough patient selection, experienced surgeons, treatment at a high-volume hospital and the implementation of an ERAS protocol morbidity and mortality can likely be reduced. Trials addressing medical or surgical interventions to reduce short-term complication are needed.

\section{Author affiliations}

${ }^{1}$ Department of Urology, Urological Research Unit, Centre for Cancer and Organ Diseases, Copenhagen University Hospital - Rigshospitalet, Copenhagen, Denmark ${ }^{2}$ Department of Clinical Medicine, University of Copenhagen, University of Copenhagen, Copenhagen, Denmark

${ }^{3}$ Department of Urology, Centre for Cancer and Organ Diseases, Copenhagen University Hospital - Rigshospitalet, Copenhagen, Denmark

${ }^{4}$ Section for Surgical Pathophysiology, The Juliane Marie Centre, Copenhagen University Hospital - Rigshospitalet, Copenhagen, Denmark

\section{Twitter Ulla Nordström Joensen @doculla}

Contributors SLM, UNJ, AMP, HK, KB and MAR contributed to the research idea and the question. SLM, UNJ and MAR wrote the protocol. SLM and MAR screened all the abstracts and full texts for inclusion. SLM did all the data extraction and statistics. SLM drafted the manuscript and UNJ, AMP, HK, KB and MAR contributed to the editing of the manuscript and approved the final manuscript.

Funding This work was supported by the Department of Urology, Rigshospitalet and Rigshospitalet's Research Committee.

Disclaimer Rigshospitalet's Research Committee did not have any role in the design, the analyses, the interpretation of data, or in the decision to submit results. Competing interests None declared.

Patient consent for publication Not required. 
Provenance and peer review Not commissioned; externally peer reviewed.

Data availability statement Data are stored and available from the corresponding author (SLM) upon reasonable request and can be reused without any further permission.

Supplemental material This content has been supplied by the author(s). It has not been vetted by BMJ Publishing Group Limited (BMJ) and may not have been peer-reviewed. Any opinions or recommendations discussed are solely those of the author(s) and are not endorsed by BMJ. BMJ disclaims all liability and responsibility arising from any reliance placed on the content. Where the content includes any translated material, BMJ does not warrant the accuracy and reliability of the translations (including but not limited to local regulations, clinical guidelines, terminology, drug names and drug dosages), and is not responsible for any error and/or omissions arising from translation and adaptation or otherwise.

Open access This is an open access article distributed in accordance with the Creative Commons Attribution Non Commercial (CC BY-NC 4.0) license, which permits others to distribute, remix, adapt, build upon this work non-commercially, and license their derivative works on different terms, provided the original work is properly cited, appropriate credit is given, any changes made indicated, and the use is non-commercial. See: http://creativecommons.org/licenses/by-nc/4.0/.

ORCID iD

Sophia Liff Maibom http://orcid.org/0000-0002-5489-2521

\section{REFERENCES}

1 Alfred Witjes J, Lebret T, Compérat EM, et al. Updated 2016 EAU guidelines on muscle-invasive and metastatic bladder cancer. Eur Urol 2017;71:462-75.

2 Moher D, Shamseer L, Clarke M, et al. Preferred reporting items for systematic review and meta-analysis protocols (PRISMA-P) 2015 statement. Syst Rev 2015;4:1.

3 Covidence. Covidence systematic review software [Internet] Veritas Health Innovation Ltd, Melbourne, Australia. Available: www. covidence.org

4 Martin RCG, Brennan MF, Jaques DP. Quality of complication reporting in the surgical literature. Ann Surg 2002;235:803-13.

5 Howick J, Chalmers I, Glasziou P. The Oxford 2011 levels of evidence". Oxford centre for evidence-based medicine. [Internet]. Available: http://www.cebm.net/index.aspx?o=5653

6 Wells G, Shea B, O'Connell D. The Newcastle-Ottawa Scale (NOS) for assessing the quality of nonrandomised studies in metaanalyses [Internet]. Available: http://www.ohri.ca/programs/clinical_ epidemiology/oxford.asp [Accessed 6 May 2020]

7 Afshar M, Goodfellow H, Jackson-Spence F, et al. Centralisation of radical cystectomies for bladder cancer in England, a decade on from the 'Improving Outcomes Guidance': the case for super centralisation. BJU Int 2018;121:217-24.

8 Al-Daghmin A, Kauffman EC, Shi Y, et al. Efficacy of robot-assisted radical cystectomy (RARC) in advanced bladder cancer: results from the International radical cystectomy Consortium (IRCC). BJU Int 2014;114:98-103.

9 Arora K, Hanson KT, Habermann EB, et al. Early complications and mortality following radical cystectomy: associations with malnutrition and obesity. Bladder Cancer 2018;4:377-88.

10 Björnsson O, Gudmundsson EO, Marteinsson VT, et al. Radical cystectomy in the treatment of bladder cancer in Iceland: a population-based study. Scand J Urol 2016;50:65-70.

11 Boorjian SA, Kim SP, Tollefson MK, et al. Comparative performance of comorbidity indices for estimating perioperative and 5-year all cause mortality following radical cystectomy for bladder cancer. $J$ Urol 2013;190:55-60.

12 Cantiello F, Cicione A, Autorino R, et al. Metabolic syndrome, obesity, and radical cystectomy complications: a clavien classification system-based analysis. Clin Genitourin Cancer 2014;12:384-93.

13 Chang SS, Cookson MS, Baumgartner RG, et al. Analysis of early complications after radical cystectomy: results of a collaborative care pathway. J Urol 2002;167:2012-6.

14 De Nunzio C, Cindolo L, Leonardo C, et al. Analysis of radical cystectomy and urinary diversion complications with the Clavien classification system in an Italian real life cohort. Eur J Surg Oncol 2013;39:792-8.

15 de Vries RR, Visser O, Nieuwenhuijzen JA, et al. Outcome of treatment of bladder cancer: a comparison between low-volume hospitals and an oncology centre. World J Urol 2010;28:431-7.
16 Djaladat H, Katebian B, Bazargani ST, et al. 90-Day complication rate in patients undergoing radical cystectomy with enhanced recovery protocol: a prospective cohort study. World J Urol 2017;35:907-11.

17 Fairey A, Chetner M, Metcalfe J, et al. Associations among age, comorbidity and clinical outcomes after radical cystectomy: results from the Alberta urology Institute radical cystectomy database. $J$ Urol 2008;180:128-34.

18 Flamiatos JF, Chen Y, Lambert WE, et al. Open versus robotassisted radical cystectomy: 30 -day perioperative comparison and predictors for cost-to-patient, complication, and readmission. $J$ Robot Surg 2019;13:129-40.

19 Gschliesser T, Eredics K, Berger I, et al. The impact of gender on tumour stage in in-house complications and choice of urinary diversion: results of the Austrian cystectomy registry. Urol Int 2017;99:429-35.

20 Hanna N, Leow JJ, Sun M, et al. Comparative effectiveness of robot-assisted vs. open radical cystectomy. Urol Oncol 2018;36:88 e1-88.e9.

21 Hayn MH, Hellenthal NJ, Hussain A, et al. Defining morbidity of robot-assisted radical cystectomy using a standardized reporting methodology. Eur Urol 2011;59:213-8.

22 Hirobe M, Tanaka T, Shindo T, et al. Complications within 90 days after radical cystectomy for bladder cancer: results of a multicenter prospective study in Japan. Int J Clin Oncol 2018;23:734-41.

23 Hollenbeck BK, Miller DC, Taub D, et al. Identifying risk factors for potentially avoidable complications following radical cystectomy. $J$ Urol 2005;174:1231-7.

24 Hollenbeck BK, Miller DC, Taub DA, et al. The effects of adjusting for case mix on mortality and length of stay following radical cystectomy. J Urol 2006;176:1363-8.

$25 \mathrm{Hu} \mathrm{JC}$, Chughtai B, O'Malley P, et al. Perioperative outcomes, health care costs, and survival after robotic-assisted versus open radical cystectomy: a national comparative effectiveness study. Eur Urol 2016;70:195-202.

26 Jerlström T, Gårdmark T, Carringer M, et al. Urinary bladder cancer treated with radical cystectomy: perioperative parameters and early complications prospectively registered in a national populationbased database. Scand J Urol 2014;48:334-40.

27 Johar RS, Hayn MH, Stegemann AP, et al. Complications after robot-assisted radical cystectomy: results from the International robotic cystectomy Consortium. Eur Urol 2013;64:52-7.

28 Johnson SC, Smith ZL, Golan S, et al. Temporal trends in perioperative morbidity for radical cystectomy using the National surgical quality improvement program database. Urol Oncol 2017;35:659.e13-659.e19.

29 Kader AK, Richards KA, Krane LS, et al. Robot-assisted laparoscopic vs open radical cystectomy: comparison of complications and perioperative oncological outcomes in 200 patients. BJU Int 2013;112:E290-4.

30 Kanno T, Ito K, Sawada A, et al. Complications and reoperations after laparoscopic radical cystectomy in a Japanese multicenter cohort. Int J Urol 2019;26:493-8.

31 Khan MS, Challacombe B, Elhage O, et al. A dual-centre, cohort comparison of open, laparoscopic and robotic-assisted radical cystectomy. Int J Clin Pract 2012;66:656-62.

32 Kim SP, Boorjian SA, Shah ND, et al. Contemporary trends of inhospital complications and mortality for radical cystectomy. BJU Int 2012;110:1163-8.

33 Koupparis A, Villeda-Sandoval C, Weale N, et al. Robot-assisted radical cystectomy with intracorporeal urinary diversion: impact on an established enhanced recovery protocol. BJU Int 2015;116:924-31.

34 Kulkarni GS, Urbach DR, Austin PC, et al. Impact of provider volume on operative mortality after radical cystectomy in a publicly funded healthcare system. Can Urol Assoc J 2013;7:425-9.

35 Lenfant L, Campi R, Parra J, et al. Robotic versus open radical cystectomy throughout the learning phase: insights from a real-life multicenter study. World J Urol 2020;38:1951-8.

36 Leow JJ, Reese S, Trinh Q-D, et al. Impact of surgeon volume on the morbidity and costs of radical cystectomy in the USA: a contemporary population-based analysis. BJU Int 2015;115:713-21

37 Liedberg F, Holmberg E, Holmäng S, et al. Long-term follow-up after radical cystectomy with emphasis on complications and reoperations: a Swedish population-based survey. Scand J Urol Nephrol 2012;46:14-18.

38 Lin W-Y, Wu C-T, Chen M-F, et al. Cystectomy for bladder cancer in elderly patients is not associated with increased 30- and 90day mortality or readmission, length of stay, and cost: propensity score matching using a population database. Cancer Manag Res 2018:10:1413-8. 
39 Liu B, Domes T, Jana K. Evaluation of an enhanced recovery protocol on patients having radical cystectomy for bladder cancer. Can Urol Assoc J 2018;12:421-6.

40 Llorente C, López B, Hernández V, et al. Variability in complications and operative mortality after radical cystectomy in Spain. Actas Urol Esp 2017;41:32-8.

41 Llorente C, Guijarro A, Hernandez V, et al. Effect of hospital volume on 90-day mortality after radical cystectomy for bladder cancer in Spain. World J Urol 2020;38:1221-8.

42 Llorente C, Guijarro A, Hernández V, et al. Outcomes of an enhanced recovery after radical cystectomy program in a prospective multicenter study: compliance and key components for success. World J Urol 2020;38:3121-9.

43 Lowrance WT, Rumohr JA, Chang SS, et al. Contemporary open radical cystectomy: analysis of perioperative outcomes. J Urol 2008;179:1313-8.

44 Malavaud B, Vaessen C, Mouzin M, et al. Complications for radical cystectomy. impact of the American Society of Anesthesiologists score. Eur Urol 2001;39:79-84.

45 Mazzone E, D'Hondt F, Beato S, et al. Robot-assisted radical cystectomy with intracorporeal urinary diversion decreases postoperative complications only in highly comorbid patients: findings that rely on a standardized methodology recommended by the European association of urology guidelines. World J Urol 2021;39:803-12.

46 Monn MF, Kaimakliotis HZ, Cary KC, et al. Short-term morbidity and mortality of Indiana pouch, ileal conduit, and neobladder urinary diversion following radical cystectomy. Urol Oncol 2014;32:1151-7.

47 Moschini M, Gandaglia G, Dell'Oglio P, et al. Incidence and predictors of 30-day readmission in patients treated with radical cystectomy: a single center European experience. Clin Genitourin Cancer 2016;14:e341-6.

48 Musch M, Janowski M, Steves A, et al. Comparison of early postoperative morbidity after robot-assisted and open radical cystectomy: results of a prospective observational study. BJU Int 2014;113:458-67.

49 Nazmy M, Yuh B, Kawachi M, et al. Early and late complications of robot-assisted radical cystectomy: a standardized analysis by urinary diversion type. J Urol 2014;191:681-7.

50 Nazzani S, Mazzone E, Preisser F, et al. Comparison of perioperative outcomes between open and robotic radical cystectomy: a population-based analysis. $J$ Endourol 2018;32:701-9.

51 Nieuwenhuijzen JA, de Vries RR, Bex A, et al. Urinary diversions after cystectomy: the association of clinical factors, complications and functional results of four different Diversions. Eur Urol 2008;53:834-44.

52 Novotny V, Froehner M, Koch R, et al. Age, American Society of Anesthesiologists physical status classification and Charlson score are independent predictors of 90-day mortality after radical cystectomy. World J Urol 2016;34:1123-9.

53 Novotny V, Hakenberg OW, Wiessner D, et al. Perioperative complications of radical cystectomy in a contemporary series. Eur Urol 2007;51:397-402.

54 Patidar N, Yadav P, Sureka SK, et al. An audit of early complications of radical cystectomy using Clavien-Dindo classification. Indian J Urol 2016;32:282-7.

55 Peyton CC, Reich RR, Tang D, et al. Identifying and Codifying complications after radical cystectomy: comparison of administrative diagnostic and procedure codes, and clinical chart review. J Urol 2019;202:913-9.

56 Porter MP, Gore JL, Wright JL. Hospital volume and 90-day mortality risk after radical cystectomy: a population-based cohort study. World J Urol 2011;29:73-7.

57 Quek ML, Stein JP, Daneshmand S, et al. A critical analysis of perioperative mortality from radical cystectomy. J Urol 2006;175:886-90.

58 Roghmann F, Sukumar S, Ravi P, et al. Radical cystectomy in the elderly: national trends and disparities in perioperative outcomes and quality of care. Urol Int 2014;92:27-34.

59 Salminen AP, Koskinen I, Perez IM, et al. Neoadjuvant chemotherapy does not increase the morbidity of radical cystectomy: a 10-year retrospective nationwide study. Eur Urol Oncol 2018;1:525-30.

60 Schmid M, Rink M, Traumann M, et al. Evidence from the 'PROspective MulticEnTer Radlcal Cystectomy Series 2011 (PROMETRICS 2011)' study: how are preoperative patient characteristics associated with urinary diversion type after radical cystectomy for bladder cancer? Ann Surg Oncol 2015;22:1032-42.
61 Shabsigh A, Korets R, Vora KC, et al. Defining early morbidity of radical cystectomy for patients with bladder cancer using a standardized reporting methodology. Eur Urol 2009;55:164-76.

62 Styn NR, Montgomery JS, Wood DP, et al. Matched comparison of robotic-assisted and open radical cystectomy. Urology 2012;79:1303-9.

$63 \mathrm{Su}$ S, Gu L, Ma X, et al. Comparison of laparoscopic and robotassisted radical cystectomy for bladder cancer: perioperative and oncologic outcomes. Clin Genitourin Cancer 2019;17:e1048-53.

64 Sung HH, Ahn J-S, Seo SI, II SS, et al. A comparison of early complications between open and robot-assisted radical cystectomy. J Endourol 2012;26:670-5.

65 Svatek RS, Fisher MB, Matin SF, et al. Risk factor analysis in a contemporary cystectomy cohort using standardized reporting methodology and adverse event criteria. J Urol 2010;183:929-34.

66 Udovicich C, Perera M, Huq M, et al. Hospital volume and perioperative outcomes for radical cystectomy: a population study. BJU Int 2017;119:26-32.

67 Wei C, Wan F, Zhao H, et al. Application of enhanced recovery after surgery in patients undergoing radical cystectomy. $J$ Int Med Res 2018;46:5011-8.

68 Wissing MD, Santos F, Zakaria AS, et al. Short- and long-term survival has improved after radical cystectomy for bladder cancer in Québec during the years 2000-2015. J Surg Oncol 2019;119:1135-44.

69 Takada N, Abe T, Shinohara N, et al. Peri-operative morbidity and mortality related to radical cystectomy: a multi-institutional retrospective study in Japan. BJU Int 2012;110:E756-64.

70 Woldu SL, Sanli O, Clinton TN, et al. Validating the predictors of outcomes after radical cystectomy for bladder cancer. Cancer 2019;125:223-31.

71 Xylinas E, Green DA, Otto B, et al. Robotic-assisted radical cystectomy with extracorporeal urinary diversion for urothelial carcinoma of the bladder: analysis of complications and oncologic outcomes in 175 patients with a median follow-up of 3 years. Urology 2013;82:1323-9.

72 Zhang JH, Ericson KJ, Thomas LJ, et al. Large single institution comparison of perioperative outcomes and complications of open radical cystectomy, Intracorporeal robot-assisted radical cystectomy and robotic extracorporeal approach. $J$ Urol 2020:203:512-21.

73 Clavien PA, Barkun J, de Oliveira ML, et al. The Clavien-Dindo classification of surgical complications: five-year experience. Ann Surg 2009;250:187-96.

74 Trotti A, Colevas AD, Setser A, et al. CTCAE v3.0: development of a comprehensive grading system for the adverse effects of cancer treatment. Semin Radiat Oncol 2003;13:176-81.

75 Hayn MH, Hellenthal NJ, Seixas-Mikelus SA, et al. Is patient outcome compromised during the initial experience with robotassisted radical cystectomy? results of 164 consecutive cases. BJU Int 2011;108:no.

76 Nutt M, Scaief S, Dynda D, et al. lleus and small bowel obstruction after radical cystectomy for bladder cancer: analysis from the nationwide inpatient sample. Surg Oncol 2018;27:341-5.

77 Cerantola Y, Valerio M, Persson B, et al. Guidelines for perioperative care after radical cystectomy for bladder cancer: enhanced recovery after surgery (ERAS $(\mathbb{R}))$ society recommendations. Clin Nutr 2013;32:879-87.

78 Pillai P, McEleavy I, Gaughan M, et al. A double-blind randomized controlled clinical trial to assess the effect of Doppler optimized intraoperative fluid management on outcome following radical cystectomy. J Urol 2011;186:2201-6.

79 Choi $\mathrm{H}$, Kang SH, Yoon DK, et al. Chewing gum has a stimulatory effect on bowel motility in patients after open or robotic radical cystectomy for bladder cancer: a prospective randomized comparative study. Urology 2011;77:884-90.

80 Lee CT, Chang SS, Kamat AM, et al. Alvimopan accelerates gastrointestinal recovery after radical cystectomy: a multicenter randomized placebo-controlled trial. Eur Urol 2014;66:265-72.

81 Inman BA, Harel F, Tiguert R, et al. Routine nasogastric tubes are not required following cystectomy with urinary diversion: a comparative analysis of 430 patients. J Urol 2003;170:1888-91.

82 Shafii M, Murphy DM, Donovan MG, et al. Is mechanical bowel preparation necessary in patients undergoing cystectomy and urinary diversion? BJU Int 2002;89:879-81.

83 Wessels F, Lenhart M, Kowalewski KF, et al. Early recovery after surgery for radical cystectomy: comprehensive assessment and meta-analysis of existing protocols. World J Urol 2020;38:3139-53.

84 Williams SB, Cumberbatch MGK, Kamat AM. Reporting radical cystectomy outcomes following implementation of enhanced 
recovery after surgery protocols: a systematic review and individual patient data meta-analysis. Eur Urol 2020:1-12.

85 Baack Kukreja JE, Messing EM, Shah JB. Are we doing "better"? The discrepancy between perception and practice of enhanced recovery after cystectomy principles among urologic oncologists. Urol Oncol 2016;34:120.e17-120.e21.

86 Clifford TG, Katebian B, Van Horn CM, et al. Urinary tract infections following radical cystectomy and urinary diversion: a review of 1133 patients. World J Urol 2018;36:775-81.

87 van Hemelrijck M, Thorstenson A, Smith P, et al. Risk of inhospital complications after radical cystectomy for urinary bladder carcinoma: population-based follow-up study of 7608 patients. BJU Int 2013;112:1113-20.

88 Kolwijck E, Seegers AEM, Tops SCM, et al. Incidence and microbiology of post-operative infections after radical cystectomy and ureteral stent removal; a retrospective cohort study. BMC Infect Dis 2019;19:303.

89 Parker WP, Tollefson MK, Heins CN, et al. Characterization of perioperative infection risk among patients undergoing radical cystectomy: results from the National surgical quality improvement program. Urol Oncol 2016;34:532.e13-532.e19.

90 Pariser JJ, Anderson BB, Pearce SM, et al. The effect of broader, directed antimicrobial prophylaxis including fungal coverage on perioperative infectious complications after radical cystectomy. Urol Oncol 2016;34:121.e9-121.e14.

91 Krasnow RE, Mossanen M, Koo S, et al. Prophylactic antibiotics and postoperative complications of radical cystectomy: a population based analysis in the United States. J Urol 2017:198:297-304.

92 European Association of Urology. Guideline on urological infections [Internet]. Available: https://uroweb.org/guideline/urologicalinfections/\#3 [Accessed 20 Apr 2020].

93 American Urological Association. Urologic procedures and antimicrobial prophylaxis, 2019. Available: https://www.auanet.org/ guidelines/urologic-procedures-and-antimicrobial-prophylaxis-( 2019) [Accessed 11 May 2020].

94 Goossens-Laan CA, Gooiker GA, van Gijn W, et al. A systematic review and meta-analysis of the relationship between hospital/ surgeon volume and outcome for radical cystectomy: an update for the ongoing debate. Eur Urol 2011;59:775-83.
95 Bruins HM, Veskimäe E, Hernández V, et al. The importance of hospital and surgeon volume as major determinants of morbidity and mortality after radical cystectomy for bladder cancer: a systematic review and recommendations by the European association of urology muscle-invasive and metastatic bladder cancer guideline panel. Eur Urol Oncol 2020;3:131-44.

96 Parekh DJ, Reis IM, Castle EP, et al. Robot-assisted radical cystectomy versus open radical cystectomy in patients with bladder cancer (razor): an open-label, randomised, phase 3, non-inferiority trial. Lancet 2018;391:2525-36.

97 Nix J, Smith A, Kurpad R, et al. Prospective randomized controlled trial of robotic versus open radical cystectomy for bladder cancer: perioperative and pathologic results. Eur Urol 2010;57:196-201.

98 Bochner BH, Dalbagni G, Sjoberg DD, et al. Comparing open radical cystectomy and robot-assisted laparoscopic radical cystectomy: a randomized clinical trial. Eur Urol 2015;67:1042-50.

99 Parekh DJ, Messer J, Fitzgerald J, et al. Perioperative outcomes and oncologic efficacy from a pilot prospective randomized clinical trial of open versus robotic assisted radical cystectomy. J Urol 2013;189:474-9.

100 Khan MS, Gan C, Ahmed K, et al. A single-centre early phase randomised controlled three-arm trial of open, robotic, and laparoscopic radical cystectomy (coral). Eur Urol 2016;69:613-21.

101 Catto JWF, Khetrapal P, Ambler G, et al. Robot-assisted radical cystectomy with intracorporeal urinary diversion versus open radical cystectomy (iROC): protocol for a randomised controlled trial with internal feasibility study. BMJ Open 2018;8:e020500-10.

102 Williams SB, Huo J, Chamie K, et al. Underutilization of radical cystectomy among patients diagnosed with clinical stage T2 muscle-invasive bladder cancer. Eur Urol Focus 2017;3:258-64.

103 Ramirez JA, Mclntosh AG, Strehlow R, et al. Definition, incidence, risk factors, and prevention of paralytic ileus following radical cystectomy: a systematic review. Eur Urol 2013;64:588-97.

104 Mitropoulos D, Artibani W, Graefen M, et al. Reporting and grading of complications after urologic surgical procedures: an AD hoc EAU guidelines panel assessment and recommendations. Eur Urol 2012;61:341-9.

105 Moher D, Liberati A, Tetzlaff J, et al. Preferred reporting items for systematic reviews and meta-analyses: the PRISMA statement. PLoS Med 2009;6:e1000097. 